\title{
Carotid artery stenting: a single-center experience
}

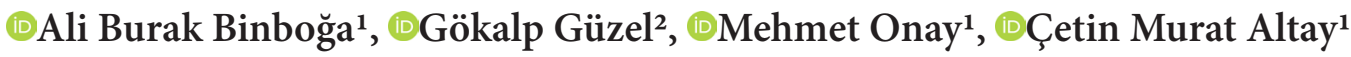 \\ ${ }^{1}$ Dr. Ersin Arslan Training and Research Hospital, Department of Radiology, Gaziantep, Turkey \\ ${ }^{2}$ Dr. Ersin Arslan Training and Research Hospital, Department of Cardiovascular Surgery, Gaziantep, Turkey
}

Cite this article as: Binboğa AB, Güzel G, Onay M, Altay ÇM. Carotid artery stenting: a single-center experience. J Health Sci Med 2022; 5(1): 138-143.

\begin{abstract}
Aim: This study aimed to present the results of carotid artery stenting (CAS) in the treatment of patients with symptomatic and asymptomatic carotid artery stenosis (Cs).

Material and Method: Between September 2016 and June 2021, patients who underwent CAS in the interventional radiology department were reviewed, retrospectively. The demographic data of patients, comorbidities, carotid stenosis rates, and early and late complication rates after treatment were recorded from medical records. Acute stent thrombosis, stroke, and death in the first 30 days after CAS were considered early periprocedural complications. Doppler ultrasonography (USG) examinations were scanned at 6 months after the procedure and restenosis rates were investigated.

Results: There were a total of 113 patients; $31.2 \%$ female and $68.8 \%$ male. Symptomatic Cs was present in $62.8 \%(\mathrm{n}=71)$ and asymptomatic Cs in $37.2 \%(\mathrm{n}=42)$ of the patients. The technical success rate was determined as $98.2 \%$. Micro-thromboembolism $(n=4)$, pseudoaneurysm at the femoral insertion site $(n=2)$, and acute stent thrombosis $(n=1)$ were observed as early complications. Restenosis was detected 6 months after stenting in 1 patient (1.4\%) with symptomatic Cs on Doppler USG. The mortality rate was $0.8 \%(n=1)$.
\end{abstract}

Conclusion: CAS is an effective and safe treatment method in symptomatic and asymptomatic patients with Cs.

Keywords: Carotid artery stenosis, carotid artery stenting, endovascular treatment, complication.

\section{INTRODUCTION}

Carotid artery stenosis (Cs) is an atherosclerotic disease that affects the extracranial carotid arteries, characterized by a decrease in lumen diameter and an increase in blood flow velocity $(1,2)$. Cs can be asymptomatic or cause a transient ischemic attack and acute ischemic stroke (2). Stroke is one of the leading causes of death and disability in adults worldwide (2). Although the prevalence of stroke in patients aged $>45$ years in Turkey varies according to region, it has been reported to be between $0.4 \%$ and $4.1 \%$ (3). Stroke is the second most common cause of death after ischemic heart disease and is responsible for $9 \%$ of all male deaths and $13 \%$ of all female deaths (4). Approximately $25-30 \%$ of all strokes are caused by Cs $(5,6)$. However, in recent years, there has been a decrease in the rate of deaths from stroke due to developments in both diagnosis and treatment methods (4).

Carotid artery stenting (CAS) and carotid artery endarterectomy (CAE) are the two main treatment modalities in the treatment of Cs. CAS is an endovascular treatment method, which has become an important alternative to CAE as a result of developments in stent technology (7-9). Due to the nature of CAS, short postoperative hospital stays and it is a minimally invasive method have made CAS more preferable. In patients with symptomatic and asymptomatic Cs, complication rates of CAS are acceptable compared to CAE. In addition, recent developments in the filter technology used to prevent stent and distal embolism, have increased the applicability of CAS more safely. However, the impact of these new developments on the periprocedural complication rates of CAS has not yet been adequately discussed.

This study aimed to present the results of CAS in the treatment of patients with symptomatic and asymptomatic Cs.

\section{MATERIAL AND METHOD}

This retrospective study was approved by Gaziantep University Clinical Researchs Ethics Committee (Date: 07.07.2021, Decision No: 2021/236). All procedures were carried out in accordance with the ethical rules and the principles of the Declaration of Helsinki. 


\section{Patients}

A retrospective review was made of patients who underwent CAS due to $\mathrm{Cs}$ in the interventional radiology clinic of Dr. Ersin Arslan Training and Research Hospital between September 2016 and March 2021. The demographic characteristics of the patients, such as age and gender, were recorded separately from the medical records. Patients with Cs who presented at the interventional radiology clinic with symptoms of transient ischemic attack (TIA), transient blindness (TB), syncope, and stroke were accepted as symptomatic Cs. The patients were separated into two groups as symptomatic Cs and asymptomatic Cs. Diagnostic digital subtraction angiography (DSA) images taken before CAS was used to diagnose Cs. The diagnosis of Cs on DSA images was made according to the criteria of "Equivalence of measurements of carotid stenosis (EMCS)" (10). Moreover, carotid plaque surfaces were classified according to DSA images as smooth plaque surface, irregular plaque surface, and plaque surface ulceration. Indications for CAS were determined according to the multi-society consensus guideline (11). In addition, comorbidities such as diabetes, hyperlipidemia, hypertension were recorded using preprocedural patient evaluation records that are routinely kept in the clinic. The technical success, complication, morbidity, and mortality rates of the procedure were investigated in patients who underwent CAS due to symptomatic and asymptomatic Cs.

\section{Carotid Artery Stenting}

Heart rate, arterial blood pressure, and oxygen saturation were monitored throughout the procedure in all patients who underwent CAS. Brain diffusion-weighted magnetic resonance imaging (DAG MRI) was performed before CAS in all patients who underwent CAS, and the presence of acute - subacute stroke was investigated. These images were also used for comparison in patients with postprocedural neurological symptoms. All patients who underwent CAS were given $75 \mathrm{mg} /$ day oral clopidogrel 5 days before the procedure or 300-450 mg oral clopidogrel was loaded 2 hours before the procedure if emergency CAS was to be performed. After the femoral

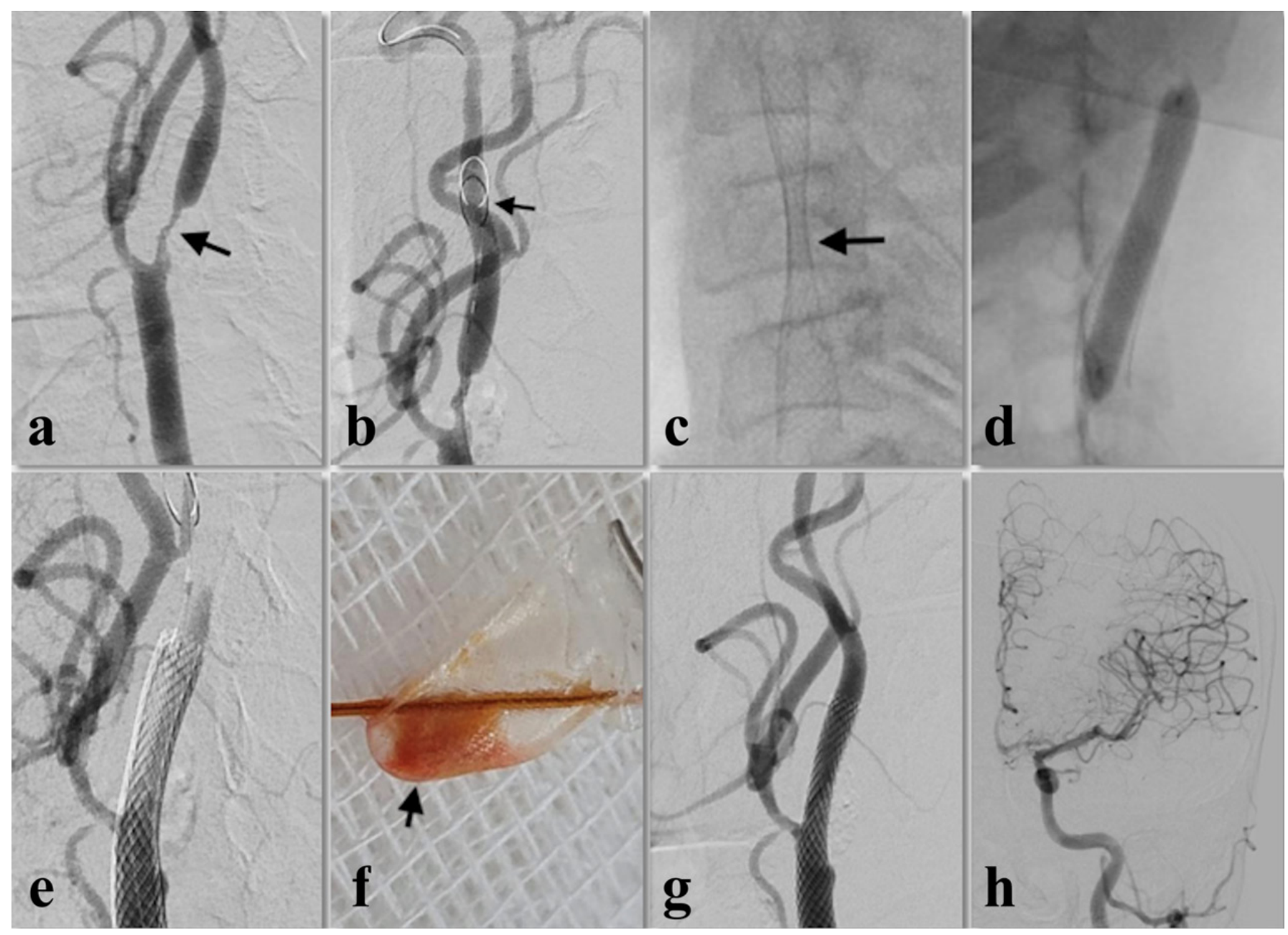

Figure 1: a-g (oblique) and h (anterior-posterior) DSA images show a 63-year-old male patient with symptomatic carotid artery stenosis. a shows long segment stenosis (black arrow). b shows the opened filter (black arrow) to prevent distal embolism. Afterward, the appropriately sized stent is opened. An hourglass appearance observes in c due to residual stenosis (black arrow). d demonstrates balloon angioplasty to obtain adequate patency. After balloon angioplasty, the control DSA image (e) is shown false occlusion in the distal ICA due to atheromatous plaques. In the same patient, the filter with atheromatous plaques is observed (f, black arrow). There is no residual stenosis (g) and no distal embolism (h). 
artery puncture during the procedure, $5000 \mathrm{IU}$ heparin was administered to maintain anticoagulation. In all patients, along vascular sheath (IVA $6 \mathrm{~F}, 80 \mathrm{~cm}$, Balt Extrusion, Montmorency, France or Neuron MAX 6F, $80 \mathrm{~cm}$, Penumbra Inc., Alameda, California, USA) was placed and cannulated from the femoral artery to the target common carotid artery (CCA). After the target lesion in the internal carotid artery (ICA) was passed with appropriate microwire manipulations, a filter was placed approximately $3 \mathrm{~cm}$ distal to the lesion to prevent distal embolism in all patients. Then, a Wallstent (Boston Scientific Inc., Watertown, Massachusetts, USA) that has a closed-cell design, of appropriate diameter and length was advanced over the filter wire and the stent was opened. The residual stenosis rate was investigated on control DSA images, and balloon angioplasty was performed in patients with stenosis $>30 \%$ to reduce the residual stenosis rate. In cases with bradycardia or a decrease in the basal heart rate of $>20 \%$ due to vagal stimulation in the carotid body during angioplasty, $1 \mathrm{mg}$ of atropine sulfate was immediately administered via the peripheral venous line. The filter was then removed and control DSA images were obtained for examination of possible thromboembolism in the intracranial arteries, and remove the filter. Then the procedure was terminated. An illustrative case was presented in Fig. 1 a-h. All patients underwent a detailed neurological examination in the angiography suite after the procedure, and the presence of neurological symptoms due to possible distal embolism was investigated. All patients were hospitalized for oneday post-procedurally and vital values were monitored. Patients with no neurological signs and stable vital values were discharged with anti-aggregant drugs (clopidogrel $75 \mathrm{mg} /$ day and acetylsalicylic acid $100 \mathrm{mg} /$ day) for at least 6 months.

\section{Follow-up}

Doppler USG was performed in all patients at 6thmonth after CAS. Restenosis was defined as $>50 \%$ Cs. For patients with neurological symptoms, DWI MRI was performed and possible CAS-induced thromboembolism was investigated by comparisons with the DWI MRI scans taken before the procedure.

\section{Statistics}

Data were analyzed using IBM SPSS Statistics v. 23 software (IBM, Armonk, NY, USA). Descriptive statistics were stated as mean \pm standard deviation (SD) for numerical variables, and as number $(\mathrm{n})$ and percentage (\%) for categorical variables. The difference between the two groups was examined with the Mann-Whitney $\mathrm{U}$ test, and the relationship between two categorical variables was examined with the Chi-Square test. A value of $\mathrm{p}<0.05$ was considered statistically significant.

\section{RESULTS}

The characteristics of the study population are summarized in Table $\mathbf{1}$. The total 113 patients comprised $31.2 \%$ females and $68.8 \%$ males, of which $62.8 \%(n=71)$ had symptomatic Cs and $37.2 \%(n=42)$ had asymptomatic Cs. The mean age of the patients was similar in symptomatic and asymptomatic groups ( $\mathrm{p}>0.05)$. The rates of the Cs plaque morphology were $48.6 \%(n=55)$ smooth surface, $33.7 \%(n=38)$ irregular surface, and $17.7 \%(n=20)$ surface ulceration. The rates of plaque surface morphology in the patient's groups were detailed in Table 1. There was a statistically significant correlation between the plaque surface classification and the patients with symptomatic Cs $(\mathrm{p}=0.016)$.

\begin{tabular}{|c|c|c|c|}
\hline Variables & $\begin{array}{c}\text { Symptomatic Cs } \\
\mathrm{n}=71(\%)\end{array}$ & $\begin{array}{c}\text { Asymptomatic Cs } \\
\mathrm{n}=42(\%)\end{array}$ & p \\
\hline Age (years) $\pm \mathrm{SD}$ & $66.06 \pm 8.22$ & $68.07 \pm 9.19$ & 0.49 \\
\hline Female & $33(46.4)$ & $22(52.3)$ & \multirow{2}{*}{0.54} \\
\hline Male & $38(53.4)$ & $20(47.7)$ & \\
\hline \multicolumn{3}{|l|}{ Stenosis rate } & \multirow[t]{3}{*}{0.67} \\
\hline $70-95 \%$ & $48(67.6)$ & $30(71.4)$ & \\
\hline $95-99 \%$ & $23(32.3)$ & $12(28.6)$ & \\
\hline \multicolumn{2}{|c|}{ Plaque morphology } & & \multirow[t]{4}{*}{$0.01^{*}$} \\
\hline Smooth & $33(46.4)$ & $22(52.3)$ & \\
\hline Irregular & $20(28.2)$ & $18(42.8)$ & \\
\hline Ulceration & $18(25.4)$ & $2(4.9)$ & \\
\hline \multicolumn{2}{|l|}{ Symptoms } & - & \multirow[t]{5}{*}{ NA } \\
\hline Stroke & \multicolumn{2}{|l|}{$31(43.6)$} & \\
\hline TIA & \multicolumn{2}{|l|}{$23(32.3)$} & \\
\hline $\mathrm{TB}$ & \multicolumn{2}{|l|}{$12(17)$} & \\
\hline Syncope & \multicolumn{2}{|l|}{$5(7.1)$} & \\
\hline HT & $58(81.6)$ & $29(69)$ & \multirow{3}{*}{0.64} \\
\hline $\mathrm{HL}$ & $50(70)$ & $19(45.2)$ & \\
\hline $\mathrm{DM}$ & $53(74.6)$ & $20(47.6)$ & \\
\hline $\mathrm{HT}+\mathrm{HL}+\mathrm{DM}$ & $42(59.1)$ & $16(38)$ & $0.03^{*}$ \\
\hline \multicolumn{4}{|c|}{$\begin{array}{l}\text { Cs: Carotid artery stenosis, TIA: Transient ischemic attack, TB: Transient blindness, } \\
\text { HT: Hypertension, HL: Hyperlipidemia, DM: Diabetes Mellitus, *: Statistically } \\
\text { significant, NA: Not Available }\end{array}$} \\
\hline
\end{tabular}

Balloon angioplasty was performed in all patients because of residual stenosis after stent placement. In patients with symptomatic Cs, the most common symptom was the stroke that was ipsilateral with stenosis. The most common comorbidity in patients with both symptomatic and asymptomatic Cs was hypertension, and this was most frequently accompanied by diabetes. No statistically significant difference was determined between the patients in both groups in terms of hypertension, hyperlipidemia, and diabetes ( $p>0.05)$. Both hypertension and diabetes were determined in $67.6 \%(n=48)$ of patients with symptomatic Cs, and in 
$35.7 \%$ of the patient group with asymptomatic Cs $(\mathrm{n}=15)$. This dual comorbidity rate was similar in both groups ( $p>0.05)$. Patients with three comorbidities (hypertension, diabetes, and hyperlipidemia) were more common in the symptomatic Cs group. Three comorbidities were determined in 59.1\% $(\mathrm{n}=42)$ of the symptomatic Cs group and $38 \%(n=16)$ of the asymptomatic Cs group $(\mathrm{p}<0.05)$.

\section{Treatment, Complications, and Follow-up Results}

Technical success was determined at the rate of $98.2 \%$. CAS could not be performed in two patients with symptomatic Cs. In one of the patients with technical failure, a filter was placed, but dense circumferential calcific plaque and lateral angulation of the ICA orifice did not allow the stent to be positioned safely in the appropriate position. In the other patient, the target artery could not be reached endovascularly, due to an anatomic variation of the right brachiocephalic trunk, which was seen to be located more inferiorly in the aortic arch. Carotid artery endarterectomy was performed in these two patients.

The complication rate in the symptomatic patient group was higher than in the asymptomatic patient group, but was not statistically significant ( $>0.05$, Table 2$)$. The complications were micro-thromboembolism $(\mathrm{n}=4)$, pseudoaneurysm at the femoral insertion site $(n=2)$, and acute stent thrombosis $(n=1)$, respectively (Table 2). Patients who experienced micro-thromboembolism intraoperatively had irregular plaque surface or plaque ulceration. Of the patients with micro-thromboembolism, 3 had weakness in the upper extremity contralateral to the lesion, and 1 had a speech impairment. Symptoms regressed in all patients with micro-thromboembolism and there was no permanent neurological deficit. When the two groups were compared in terms of microthromboembolism, there was no statistically significant difference $(p=0.608)$. One of the two patients with pseudoaneurysm was treated percutaneously and the other was treated surgically. The patient with acute stent thrombosis presented at the Emergency Department with hemiplegia on the 7 th day after CAS. Thrombus causing total occlusion in the stent was detected on Doppler USG, and it was learned that the patient had stopped taking the anti-aggregant drugs. This patient died after intensive care follow-up. All patients who underwent CAS for both symptomatic and asymptomatic Cs had a 6-month follow-up Doppler USG. Restenosis was detected 6 months after stenting in 1 patient $(1.4 \%)$ with symptomatic Cs on follow-up Doppler USG. The rate of restenosis in this patient was between $50-69 \%$, and as the patient was asymptomatic, a revision procedure was not considered.

\begin{tabular}{|c|c|c|c|}
\hline Variables & $\begin{array}{c}\text { Symptomatic } \\
\text { Cs } \\
n=69^{*}(\%) \\
\end{array}$ & $\begin{array}{c}\text { Asymptomatic } \\
\text { Cs } \\
\mathrm{n}=42(\%)\end{array}$ & $\mathbf{p}$ \\
\hline Technical success rates & $97.1 \%$ & $\% 100$ & NA \\
\hline Complications & & & 0.62 \\
\hline Microembolism & $3(4.3)$ & $1(2.4)$ & \\
\hline Acute stent thrombosis & $1(1.4)$ & - & \\
\hline Pseudoaneurysm & $1(1.4)$ & $1(2.4)$ & \\
\hline None & $66(92.9)$ & $40(95.2)$ & \\
\hline Restenosis & & & NA \\
\hline Yes & $1(1.4)$ & 0 & \\
\hline No & $68(98.5)$ & $42(100)$ & \\
\hline
\end{tabular}

\section{DISCUSSION}

Cs causes $15-20 \%$ of all ischemic strokes (12). Severe CAS of $70-99 \%$ stenosis can lead to recurrent ischemic events (12). Therefore, patients who are symptomatic or asymptomatic but with severe stenosis are Candidates for revascularization treatments such as CAS or CAE (13). In a systematic review of guidelines for the management of asymptomatic carotid stenosis, CAS can be performed in asymptomatic patients with moderate-severe Cs (5099\% stenosis) to prevent future ischemic events (13). In our study, all asymptomatic patients had 70-99\% stenosis. While CAE is a surgical treatment, CAS is a minimally invasive endovascular method. CAS has been performed more frequently than CAE in recent years, as a result of developments in-stent and catheter technology. Many studies in the literature have compared the results of CAS and CAE. The Carotid Revascularization Endarterectomy vs. Stenting Trial (CREST) study reported the 4-year followup results of randomized CAS and CAE in 2502 patients with symptomatic or asymptomatic Cs (9). According to the results of the CREST study, it was stated that the rates of stroke and death after the procedure were higher in both symptomatic and asymptomatic patients in the CAS group than in the CAE group (9). In a randomized study comparing rates of ischemia in patients with symptomatic Cs, there was reported to be no difference between CAS and CAE (14). Recent meta-analyses have stated similar postprocedural complication rates for both CAS and $\operatorname{CAE}(15,16)$. CAS is an effective treatment option in the prevention of stroke, which may cause long-term mortality or morbidity (12). Developments in-stent and catheter technology and increased operator experience may have led to better results in CAS in recent years. In markets opencell design and closed-cell design stents were available. These two different stent cell designs exhibit clinical significance in terms of complication. Stabile et al. (17) investigated the impact of different types of carotid stents on clinical outcomes in 1,604 patients. They emphasized open-cell design may be associated with an increased 
30-day stroke risk (17). Because atherosclerotic plaque components and microthrombosis can be jailed between the stent wall and carotid artery intima by closed-cells of the stent. In our department, we use a closed-cell design stent to decrease 30-day stroke risk. Symptomatic patients were more common in the current study population (Table 1). The most common symptoms of Cs are stroke, TIA, and TB (9). In this study, the most common symptom was a stroke, which was consistent with the literature. It is important that patients with Cs are symptomatic before the procedure because the most important factor affecting the postoperative complication rates of CAS is whether the patient is symptomatic or asymptomatic before stenting $(9,15,16)$. Plaque morphology affects both whether the patient is symptomatic or not and the occurrence of intraoperative complications. Plaque stability and morphology links with plaque histopathology (18). The majority of stable plaque has a thick fibrous cap and exhibits low intraoperative complication rates (18). On the other hand, unstable plaques are more prone to embolic complications (18). Choi et al. (19) noted that plaque surface morphology on DSA was a highly sensitive marker of plaque instability during CAS. In our study, the patients with symptomatic Cs had irregular plaque surface and plaque ulceration more frequently than the patients with asymptomatic Cs. In addition, intraoperative complications were common in patients with irregular plaque surface and plaque ulceration. Our data were compatible with the literature.

Complication rates may increase in patients with symptomatic Cs. Vatan et al. (20) reported that the most common periprocedural complication of CAS is ischemic events. Roubin et al. (21) reported the rate of major stroke as $1 \%$ and the rate of minor stroke as $4.8 \%$ after CAS. In the current study, the rate of minor stroke was $4.3 \%$ in patients with symptomatic Cs and $2.4 \%$ in asymptomatic patients, which is consistent with findings in the literature. Although the rate of minor stroke in patients with symptomatic Cs was proportionally higher than in asymptomatic patients in this study, it did not reach statistical significance. Further studies with more patients are needed.

Another complication associated with stenting is acute stent thrombosis, which is a rare but fatal complication of CAS. According to the time of carotid stent thrombosis, it is seen in 3 different periods as acute (first 30 days after CAS), late (after 30 days), and very late (after 1 year) (22). Anti-aggregant medication is one of the most important factors in the prevention of stent thrombosis because, during stenting, damage occurs in atheroma plaque and the target artery endothelium $(22,23)$. Therefore, anti-aggregant medication is routinely recommended to all our patients undergoing CAS for the prevention of stent thrombosis. One patient in the current study had acute stent thrombosis, which was fatal due to the discontinuation of the anti-aggregant medication. This finding may emphasize the importance of anti-aggregant medication in line with the literature.

Stenting may also cause a foreign body reaction, and restenosis may occur as a result of wound healing reactions (24). Restenosis rates after CAS have been reported as $11.1 \%$ (25). In the current study, only one patient in the CAS group had restenosis at 6 months and did not require treatment. There may be several reasons why the current study results seem better than previous findings in the literature. There may have been an effect from the type of stent used and there could be differences in patient compliance with anti-aggregant medication. More comprehensive and comparative studies with more patients are needed.

The technical success rates of CAS vary according to the vascular anatomy of the patients. In particular, anatomical variations in the vascular structures from the groin to the target ICA or the tortuous course can have significant effects. Müller et al. (16) reported that the complex configuration of the aortic arch and the presence of wide-angle CCA affect technical success in patients undergoing CAS. A complex aortic arch and wide CCA angulation have been stated as risk factors for ischemic complications. In the current study, technical failure occurred in one patient due to challenging vascular anatomy. In another patient, the intense calcific load of the atherosclerotic plaque caused by Cs, wide CCA angulation, and low stent flexibility can be said to be the reasons for technical failure.

This study had some limitations, primarily the retrospective and single-center design. Another limitation was the relatively low sample size, which limited the interpretation of statistical data. Moreover, DSA could be performed in patients with re-stenosis to confirm the severity of the stenosis. But, Doppler US usually may be a sufficient examination for patients with re-stenosis. However, these limitations were not considered to detract from the value of the study as there are so few studies discussing the outcome of CAS in patients with symptomatic and asymptomatic Cs. Therefore, this study can be considered to contribute to the literature.

\section{CONCLUSION}

CAS is an effective and safe treatment option in symptomatic and asymptomatic patients with Cs. Although not at a statistically significant level, there tended to be fewer complications of CAS in patients with asymptomatic Cs compared to patients with symptomatic Cs. Nevertheless, there is a need for further studies involving more patients. 


\section{ETHICAL DECLARATIONS}

Ethics Committee Approval: This retrospective study was approved by Gaziantep University Clinical Researchs Ethics Committee (Date: 07.07.2021, Decision No: 2021/236).

Informed Consent: Because the study was designed retrospectively, no written informed consent form was obtained from patients.

Referee Evaluation Process: Externally peer-reviewed.

Conflict of Interest Statement: The authors have no conflicts of interest to declare.

Financial Disclosure: The authors declared that this study has received no financial support.

Author Contributions: All of the authors declare that they have all participated in the design, execution, and analysis of the paper, and that they have approved the final version.

\section{REFERENCES}

1. Saxena A, Ng EYK, Lim ST. Imaging modalities to diagnose carotid artery stenosis: progress and prospect. Biomed Eng 2019; 18: 1-23.

2. Krist AH, Davidson KW, Mangione CM, et al. Screening for asymptomatic carotid artery stenosis: US preventive services Task force recommendation statement. JAMA 2021; 325: 476-81.

3. Türk Börü Ü, Kulualp AŞ, Tarhan ÖF, et al. Stroke prevalence among the Turkish population in a rural area of Istanbul: A community-based study. SAGE Open Med 2018; 6: 1-6.

4. Soto Á, Guillén-Grima F, Morales G, Muñoz S, Aguinaga-Ontoso I. Trends in mortality from stroke in the European Union. Eur J Neurol 2021; 28: 182-91.

5. Ertaş F, Çevik MU, Aluçlu MU, et al. Karotis arter stentlemesi: Ínvaziv bir üçüncü basamak merkez deneyiminin retrospektif değerlendirilmesi. Dicle Med J 2016; 43: 141-5.

6. Onal Y, Velioglu M, Demir U, Celikoglu E, Karakas HM. Feasibility of distal mechanical thrombectomy in $\mathrm{m} 3, \mathrm{a} 3$, and $\mathrm{p} 3$ segments via a 0.013 -inch delivery system: preliminary experience. Turk Neurosurg 2020; 30: 614-20.

7. Brott TG, Hobson RW, Howard G, et al. Stenting versus endarterectomy for treatment of carotid-artery stenosis. N Engl J Med 2010; 363: 11-23.

8. Silver FL, Mackey A, Clark WM, et al. Safety of stenting and endarterectomy by symptomatic status in the Carotid Revascularization Endarterectomy Versus Stenting Trial (CREST). Stroke 2011; 42: 675-80.

9. Timaran CH, Mantese VA, Malas M, et al. Differential outcomes of carotid stenting and endarterectomy performed exclusively by vascular surgeons in the Carotid Revascularization Endarterectomy versus Stenting Trial (CREST). J Vasc Surg 2013; 57: 303-8.

10.Saba L, Mallarini G. A comparison between NASCET and ECST methods in the study of carotids: evaluation using Multi-DetectorRow CT angiography. Eur J Radiol 2010; 76: 42-7.

11. Brott TG, Halperin JL, Abbara S, et al. 2011 ASA/ACCF/AHA/ AANN/AANS/ACR/ASNR/CNS/SAIP/SCAI/SIR/SNIS/SVM/ SVS Guideline on the Management of Patients With Extracranial Carotid and Vertebral Artery Disease: Executive Summary. Circulation 2011; 124: 489-532.
12. Wabnitz AM, Turan TN. Symptomatic carotid artery stenosis: surgery, stenting, or medical therapy? Curr Treat Options Cardiovasc Med 2017; 19: 1-12.

13. The SPACE Collaborative Group. 30 day results from the SPACE trial of stent-protected angioplasty versus carotid endarterectomy in symptomatic patients: a randomised non-inferiority trial. Lancet 2006; 368: 1239-47.

14. Sardar P, Chatterjee S, Aronow HD, et al. Carotid artery stenting versus endarterectomy for stroke prevention: a meta-analysis of clinical trials. JACC 2017; 69: 2266-75.

15. Müller MD, Lyrer P, Brown MM, Bonati LH. Carotid artery stenting versus endarterectomy for treatment of carotid artery stenosis. Cochrane Database Syst Rev 2020.

16. Stabile E, Giugliano G, Cremonesi A, et al. Impact on outcome of different types of carotid stent: results from the European Registry of Carotid Artery Stenting. EIJ 2016; 12: 265-70.

17. Brinjikji W, Huston J, Rabinstein AA, Kim G-M, Lerman A, Lanzino G. Contemporary carotid imaging: from degree of stenosis to plaque vulnerability. J Neurosurg 2016; 124: 27-42.

18. Choi J-H, Park H-S, Kim D-H, Cha J-K, Huh J-T, Kang M. Direct relationship between angiographic characteristics of carotid atherosclerotic plaque and filling defect in the cerebral protection filters: based on the conventional angiography. J Korean Neurosurg Soc 2013; 54: 93.

19. Vatan MB, Acar AB, Aksoy M, et al. Predictors of periprocedural complications of carotid artery stenting - a multivariate analysis of a single-centre experience. Vasa 2016; 45: 387-93.

20. Roubin GS, New G, Iyer SS, et al. Immediate and late clinical outcomes of carotid artery stenting in patients with symptomatic and asymptomatic carotid artery stenosis: a 5-year prospective analysis. Circulation 2001; 103: 532-7.

21. Moulakakis KG, Mylonas SN, Lazaris A, et al. Acute carotid stent thrombosis: a comprehensive review. Vasc Endovascular Surg 2016; 50: 511-21.

22. Texakalidis P, Giannopoulos S, Jonnalagadda AK, et al. Carotid artery endarterectomy versus carotid artery stenting for restenosis after carotid artery endarterectomy: a systematic review and meta-analysis. World Neurosurg 2018; 115: 421-9.

23. Stilo F, Montelione N, Calandrelli R, et al. The management of carotid restenosis: a comprehensive review. Ann Transl Med 2020; 8: 1272 .

24. Eckstein HH, Ringleb P, Allenberg JR, et al. Results of the StentProtected Angioplasty versus Carotid Endarterectomy (SPACE). study to treat symptomatic stenoses at 2 years: a multinational, prospective, randomised trial. Lancet Neurol 2008; 7: 893-902. 